Research Article

\title{
Formation of 2s-State Hydrogen Atom in Proton-Lithium Inelastic Scattering
}

\author{
S. A. Elkilany ${ }^{1,2}$ \\ ${ }^{1}$ Department of Mathematics, College of Science, Kafrelsheikh University, Kafrelsheikh, Egypt \\ ${ }^{2}$ Department of Mathematics, College of Science, Ad Dammam University, P.O. Box 838, Ad Dammam, 31113, Saudi Arabia
}

Correspondence should be addressed to S. A. Elkilany; sabbelkilany@yahoo.com

Received 21 January 2014; Accepted 24 March 2014; Published 6 May 2014

Academic Editor: John R. Sabin

Copyright (c) 2014 S. A. Elkilany. This is an open access article distributed under the Creative Commons Attribution License, which permits unrestricted use, distribution, and reproduction in any medium, provided the original work is properly cited.

\begin{abstract}
Elaborate coupled static formalism is employed for treatment of proton-lithium collisions at wide range of incident energies between 10 and $1000 \mathrm{Kev}$. Coupled static and frozen core approximations are employed for calculating partial and total cross sections. Only elastic and formation of excited hydrogen, $\mathrm{H}(2 \mathrm{~s})$, channels are considered. Total cross sections are calculated using seven partial waves Green's function expansion technique of total angular momentum $\ell(0 \leq \ell \leq 6)$. Proposed iterative approach allows for reliable representation of the core potentials using elaborate variational calculation of target orbitals. Polarization potential of lithium atom is taken into consideration in calculating corresponding total cross sections. Quite interesting reliable results were obtained in comparison with other theoretical approaches.
\end{abstract}

\section{Introduction}

There has been a growing interest in the investigation of electron capture from alkali-metal atom. As an example, charge-transfer process with Li has been suggested to occur in plasma diagnostic probes [1]. Besides, alkali-metal atoms are many-electron systems that can be simplified to be oneelectron systems due to a single valence electron. Theoretical calculations as well as experimental measurements have been carried out for ionization of and electron capture from alkali-metal atoms by proton impact $[1,2]$. Investigation of elastic scattering of positrons from noble gases using an iterative Green's function partial wave expansion formalism and a model static and polarization potentials are derived for describing the interactions of positrons with closed shell atoms $[3,4]$. The formation of excited hydrogen atom $(\mathrm{H}(2 \mathrm{~s}))$ in the process $\mathrm{H}^{+}+\mathrm{Li} \rightarrow \mathrm{H}(2 \mathrm{~s})+\mathrm{Li}^{+}$has been studied only in low energy ranges. The electron capture cross sections from Li by high energy (for heavy particle collision on atoms, the ratio of the projectile velocity to the orbital electron velocity is much larger than 1) incident protons in energy range between 200 and $10000 \mathrm{Kev}$ have been investigated by using the continuum distorted wave (CDW) approximation [5].
The formation of excited hydrogen atom $(\mathrm{H}(2 \mathrm{~s}))$ in Oppenheimer Brinkman and Kramer (OBK) approximation is investigated [6]. The differential and total cross sections have been investigated in the formation of $\mathrm{H}$-atom in the 2s-excited state of proton-lithium scattering by using the Coulomb projectile Born (CPB) approximation in the energy range from 50 to $10000 \mathrm{Kev}$ [7]. Proton-alkali atom ( Na, K, $\mathrm{Rb}$, and $\mathrm{Cs}$ ) collision has also studied in the wave formulation of impulse approximation in the energies ranging from 50 to $500 \mathrm{Kev}$ [8]. The polarized orbital method (POM) has been applied to study elastic scattering of positrons by large atoms, finding that this method is extremely successful in the treatment of elastic scattering of positrons by noble gases [9]. The POM yields a considerable improvement in static phase shifts which were always shifted towards the variational ones [10]. The improvement was due to the effect of the polarization potentials which play an important role in the real physical picture of the considered collision process.

In this work, an elaborate coupled static formalism is employed [11] for the treatment of proton-lithium collisions at wide range of incident energies from 10 to $1000 \mathrm{Kev}$. The proposed iterative approach allows for reliable representation of the core potentials using elaborate variational calculation 


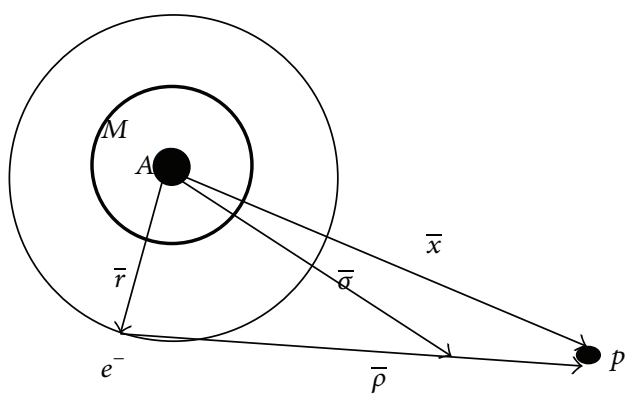

FIgURE 1: Configuration space of $p$-Li scattering.

of the target orbitals. The effect of "switching on" polarization potential of lithium atom on their coupled static cross sections is investigated. We assume that the elasticity and the formation of $\mathrm{H}(2 \mathrm{~s})$ channels are open and that all other channels of target are closed. Quite interesting reliable results were obtained in comparison with other theoretical approaches.

\section{Theoretical Formalism}

The two-channel scattering problems under investigation can be sketched by [11]

$$
p+\operatorname{Li}\{2 s\}= \begin{cases}p+\operatorname{Li}\{2 s\} & (\text { elastic channel) } \\ \mathrm{H}\{2 \mathrm{~s}\}+\mathrm{Li}^{+} & (\mathrm{H}(2 \mathrm{~s}) \text { formation channel })\end{cases}
$$

The total Hamiltonian of the first channel, elastic channel (in Rydberg units and frozen core approximation), has the following form:

$$
\begin{aligned}
H & =H^{(1)}=H_{\mathrm{Li}(2 \mathrm{~s})}-\frac{1}{2 \mu_{M}} \nabla_{x}^{2}+V_{\mathrm{int}}^{(1)} \\
& =-\frac{1}{2 \mu_{m}} \nabla_{r}^{2}-\frac{2}{r}-\frac{1}{2 \mu_{M}} \nabla_{x}^{2}+V_{\mathrm{int}}^{(1)},
\end{aligned}
$$

where $\mu_{m}, \mu_{M}$ are reduced masses of the target and first channel, respectively. $\bar{x}$ and $\bar{r}$ are the position vectors of projectile proton and valence electron of target with respect to center of mass of target, $\bar{\rho}$ is the position vector of projectile proton with respect to valence electron of target, and $\bar{\sigma}$ is the position vector of center of mass of $\mathrm{H}(2 \mathrm{~s})$ from target nucleus (see Figure 1). $M_{A}$ is mass of the nucleus of target and $V_{\text {int }}^{(1)}$ denotes the interaction between the incident proton and target atom; that is,

$$
V_{\text {int }}^{(1)}=\frac{2}{x}-\frac{2}{|x-r|}+V_{c \text { Coul }}(x)
$$

where

$$
\begin{gathered}
V_{c \text { Coul }}(x)=-\left.V_{c \text { Coul }}(r)\right|_{r=x}, \\
V_{c \text { Coul }}(r)=\left\langle\Phi_{\mathrm{Li}(1 \mathrm{~s})}\left(r_{i}\right)\left|\frac{2}{\left|r-r_{i}\right|}-\frac{2}{r}\right| \Phi_{\mathrm{Li}(1 \mathrm{~s})}\left(r_{i}\right)\right\rangle,
\end{gathered}
$$

and the corresponding total energy in first channel is determined by

$$
E=E^{(1)}=E_{\mathrm{Li}(2 s)}+\frac{1}{2 \mu_{M}} k_{1}^{2},
$$

where $\left(1 / 2 \mu_{M}\right) k_{1}^{2}$ is the kinetic energy of incident proton relative to target nucleus. $\left|\Phi_{\mathrm{Li}(1 \mathrm{~s})}\left(r_{i}\right)\right\rangle, i=1,2$, is the wave function of $i$ th electron in 1s-orbital and $\left|\Phi_{\mathrm{Li}(2 \mathrm{~s})}(r)\right\rangle$ is the wave function of valence electron. They are expanded following Clementi and Roetti [12] and adjusted such that the binding energy of the valence electron $E_{\mathrm{Li}(2 \mathrm{~s})}$ is minimum; that is,

$$
E_{\mathrm{Li}(2 s)}=\left\langle\Phi_{\mathrm{Li}(2 s)}(r)\left|H_{\mathrm{Li}(2 s)}\right| \Phi_{\mathrm{Li}(2 \mathrm{~s})}(r)\right\rangle .
$$

The total Hamiltonian of the second channel, $\mathrm{H}(2 \mathrm{~s})$ formation, is expressed (in Rydberg units and frozen core approximation) as

$$
\begin{aligned}
H & =H^{(2)}=H_{\mathrm{H}(2 \mathrm{~s})}-\frac{1}{2 \mu_{M^{\prime}}} \nabla_{\sigma}^{2}+V_{\mathrm{int}}^{(2)} \\
& =-\frac{1}{2 \mu_{m^{\prime}}} \nabla_{\rho}^{2}-\frac{2}{\rho}-\frac{1}{2 \mu_{M^{\prime}}} \nabla_{\sigma}^{2}+V_{\mathrm{int}}^{(2)},
\end{aligned}
$$

where $\mu_{m^{\prime}}, \mu_{M^{\prime}}$ are reduced masses of $\mathrm{H}(2 \mathrm{~s})$ and the second channel, respectively. $V_{\text {int }}^{(2)}$ represents the interaction between the particles of $\mathrm{H}(2 \mathrm{~s})$ and rest of target atom; that is,

$$
V_{\text {int }}^{(2)}=\frac{2}{x}-\frac{2}{r}+V_{c \text { Coul }}(x)+V_{c \text { Coul }}(r)+V_{c e x}(r),
$$

where

$$
V_{c e x}(r)=\left\langle\Phi_{\mathrm{Li}(2 \mathrm{~s})}\left(r_{i}\right)\left|\frac{2}{\left|r-r_{i}\right|}-\frac{2}{r}\right| \Phi_{\mathrm{Li}(2 \mathrm{~s})}\left(r_{i}\right)\right\rangle,
$$

and the total energy of second channel is determined by

$$
E=E^{(2)}=E_{\mathrm{H}(2 \mathrm{~s})}+\frac{1}{2 \mu_{M^{\prime}}} k_{2}^{2},
$$

where $\left(1 / 2 \mu_{M^{\prime}}\right) k_{2}^{2}$ is the kinetic energy of center of mass of $\mathrm{H}(2 \mathrm{~s})$ with respect to nucleus of target. It is related to the energy of the incident proton by

$$
\frac{k_{2}^{2}}{2 \mu_{M^{\prime}}}=\left(E_{\mathrm{Li}(2 s)}+\frac{1}{2 \mu_{M}} k_{1}^{2}-E_{\mathrm{H}(2 s)}\right),
$$

where $E_{\mathrm{H}(2 \mathrm{~s})}=-0.25 \mathrm{Ry}$ is the ground state energy of $\mathrm{H}(2 \mathrm{~s})$ and $k_{2}^{2} / 2 \mu_{M^{\prime}}>0$ means that $\mathrm{H}(2 \mathrm{~s})$ channel is open; otherwise, it is closed. Thus, $\mathrm{H}(2 \mathrm{~s})$ formation is only possible if $k_{1}^{2}>2 \mu_{M}\left(E_{\mathrm{H}(2 \mathrm{~s})}-E_{\mathrm{Li}(2 \mathrm{~s})}\right)$.

The coupled static approximation states that the solution of the two-channel scattering problem under consideration is subjected to the following conditions [11]:

$$
\begin{aligned}
& \left\langle\Phi_{\mathrm{Li}(2 s)}|H-E| \Psi\right\rangle=0, \\
& \left\langle\Phi_{\mathrm{H}(2 \mathrm{~s})}|H-E| \Psi\right\rangle=0,
\end{aligned}
$$


where $|\Psi\rangle$ is the total wave function describing each scattering process; that is,

$$
|\Psi\rangle=\left|\Phi_{\mathrm{Li}(2 \mathrm{~s})}(r)\right\rangle\left|\psi_{1}(x)\right\rangle+\left|\Phi_{\mathrm{H}(2 \mathrm{~s})}(\rho)\right\rangle\left|\psi_{2}(\sigma)\right\rangle,
$$

where $\Phi_{\mathrm{H}(2 \mathrm{~s})}=(1 / \sqrt{32 \pi})(2-\rho) \exp (-\rho / 2)$ is the ground state wave function of $\mathrm{H}(2 \mathrm{~s}), \psi_{1}(x)$ is the wave function describing scattered protons, and $\psi_{2}(x)$ is the scattering wave function of second channel.

Substituting (3)-(5) in (2), we obtain

$$
\begin{aligned}
& \frac{1}{2 \mu_{M}}\left(\nabla_{x}^{2}+k_{1}^{2}\right)\left|\psi_{1}\right\rangle \\
& \quad=U_{s t}^{(1)}(x)\left|\psi_{1}\right\rangle+\left\langle\Phi_{\mathrm{Li}(2)}\left|H^{(2)}-E^{(2)}\right| \Phi_{\mathrm{H}(2 s)} \psi_{2}\right\rangle .
\end{aligned}
$$

Substituting (4) and (8)-(10) in (7), we obtain

$$
\begin{aligned}
& \frac{1}{2 \mu_{M^{\prime}}}\left(\nabla_{\sigma}^{2}+k_{2}^{2}\right)\left|\psi_{2}\right\rangle \\
& \quad=U_{s t}^{(2)}(\sigma)\left|\psi_{2}\right\rangle+\left\langle\Phi_{\mathrm{H}(2 \mathrm{~s})}\left|H^{(1)}-E^{(1)}\right| \Phi_{\mathrm{Li}(2 \mathrm{~s})} \psi_{1}\right\rangle,
\end{aligned}
$$

where Schrödinger's equations of the target and $\mathrm{H}(2 \mathrm{~s})$ are employed. The potentials $U_{s t}^{(1)}(x)$ and $U_{s t}^{(2)}(\sigma)$ are defined by

$$
\begin{aligned}
& U_{s t}^{(1)}(x)=\left\langle\Phi_{\mathrm{Li}(2 s)}(r)\left|V_{\mathrm{int}}^{(1)}\right| \Phi_{\mathrm{Li}(2 s)}(r)\right\rangle, \\
& U_{s t}^{(2)}(\sigma)=\left\langle\Phi_{\mathrm{H}(2 s)}(r)\left|V_{\mathrm{int}}^{(2)}\right| \Phi_{\mathrm{H}(2 \mathrm{~s})}(r)\right\rangle .
\end{aligned}
$$

In order to test the effect of polarization of lithium, we switch on the polarization potential of lithium atom, that is, $V_{\text {pol }}^{\mathrm{Li}(2 s)}(\sigma)$. This can be done by replacing $U_{s t}^{(2)}(x)$ by a potential $U^{(2)}(x)$ such that

$$
U^{(2)}(x)=U_{s t}^{(2)}(\sigma)+\lambda_{1} V_{\text {pol }}^{\mathrm{Li}(2)}(\sigma),
$$

where $\lambda_{1}=1$ if the polarization is switched on (Model II) and $\lambda_{1}=0$ otherwise (Model I). We choose $V_{\text {pol }}^{\mathrm{Li}(2)}(\sigma)$ in the Peach form [13],

$$
V_{\text {pol }}^{\mathrm{Li}(2 \mathrm{~s})}(x)=-\frac{\alpha}{x^{4}}\left\{1.0-\left(1.0+\gamma x+\frac{(\gamma x)^{2}}{2}\right) e^{-\gamma x}\right\},
$$

where $\alpha=0.19$ and $\gamma=3.91$.

Using partial wave expansions of the scattering wavefunctions $\left|\psi_{1}\right\rangle$ and $\left|\psi_{2}\right\rangle$ in (14) and (15) that solutions are given (formally) by Lippmann-Schwinger equation [14]

$$
|\xi\rangle=\left|\xi_{0}\right\rangle+G_{0}|\zeta\rangle
$$

where $G_{0}$ is Green operator $\left(E-H_{0}\right)^{-1}$ and $\left|\xi_{0}\right\rangle$ is solution of the homogeneous equation:

$$
\left(E-H_{0}\right)\left|\xi_{0}\right\rangle=|0\rangle .
$$

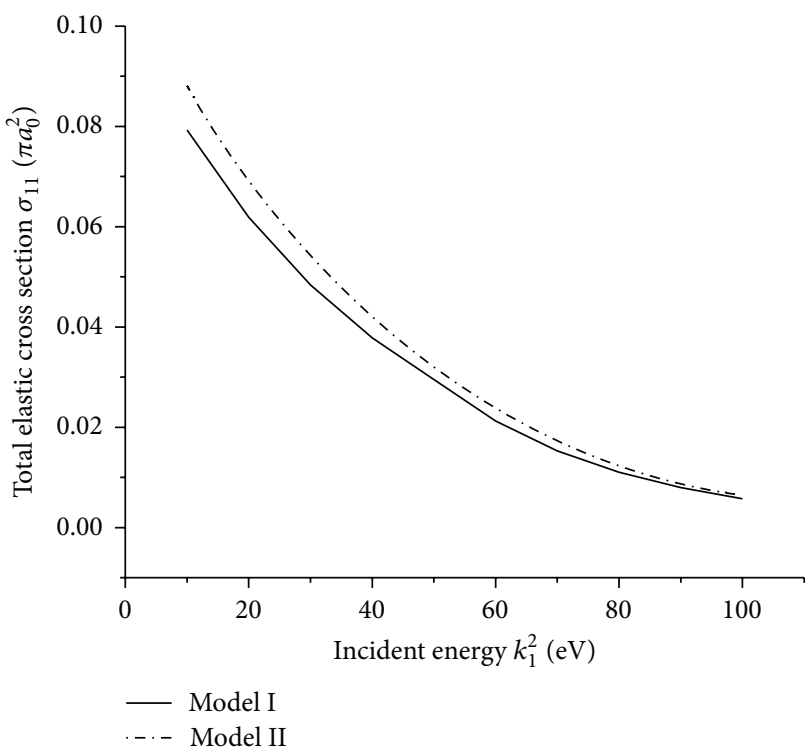

FIGURE 2: Total elastic cross sections $\left(\sigma_{11}\right.$ in $\left.\pi a_{0}^{2}\right)$ of proton-lithium scattering by coupled static approximation for the two models.

The partial wave expansions of Green operators corresponding to operators on the two differential equations enable us to write their solutions in integral form that can be solved by iterative numerical technique; then, we obtain reactance matrix $R^{v}$ that is related to transition matrix $T^{v}$ by

$$
T^{\nu}=R^{\nu}(I-\widetilde{i} R)^{-1},
$$

where $v$ is order of iteration, $I$ is a $2 \times 2$ unit matrix, and $\tilde{i}=$ $\sqrt{-1}$. Partial cross sections obtained in an iterative way are determined (in $\pi a_{0}^{2}$ units) using the relation

$$
\sigma_{i j}^{(\ell, v)}=\frac{4(2 \ell+1)}{k_{i}^{2}}\left|T_{i j}^{\nu}\right|^{2}, \quad i=1,2 .
$$

Finally, the total cross sections (in $\pi a_{0}^{2}$ units) are expressed (in $\nu$ th iteration) by

$$
\sigma_{i j}^{v}=\sum_{\ell=0}^{\infty} \sigma_{i j}^{(\ell, v)}, \quad i, j=1,2, \nu>0 .
$$

\section{Results and Discussion}

We start our investigation by testing the variation of static potentials of two channels, $U_{s t}^{(1)}(x)$ and $U_{s t}^{(2)}(\sigma)$, and the polarization potential $V_{\text {pol }}^{\mathrm{Li}(2 \mathrm{~s})}(\sigma)$ of lithium atom, with increase of $\sigma$ and $x$. Values of $\sigma$ and $x$ have been chosen such that $\sigma=$ $x=1 / 16,2 / 16,3 / 16, \ldots, 512 / 16$ where $h=1 / 16$ is the mesh size (or Simpson's step) employed for calculating integrals appearing in integral equations using Simpson's rule.

Calculation of cross sections of proton-lithium ( $p$-Li) scattering has been proceeded by investigating variation of elements of $R^{v}$ with increase of integration range (IR) and 
TABLE 1: Partial and total elastic cross sections $\left(\sigma_{11}\right.$ in $\left.\pi a_{0}^{2}\right)$ of proton-lithium scattering by coupled static approximation. Integration range $\left(\mathrm{IR}=32 a_{o}\right)$, number of iterations $(v=50)$ (Model I).

\begin{tabular}{lcccccccc}
\hline$k^{2} \mathrm{kev}$ & $L=0$ & $L=1$ & $L=2$ & $L=3$ & $L=4$ & $L=5$ & $L=6$ & Total \\
\hline 10 & $4.7641 E-02$ & $1.9057 E-02$ & $7.6225 E-03$ & $3.0490 E-03$ & $1.2196 E-03$ & $4.8784 E-04$ & $1.9514 E-04$ & $7.9271 E-02$ \\
20 & $3.7220 E-02$ & $1.4888 E-02$ & $5.9550 E-03$ & $2.3820 E-03$ & $9.5283 E-04$ & $3.8112 E-04$ & $1.5245 E-04$ & $6.1931 E-02$ \\
30 & $2.9077 E-02$ & $1.1631 E-02$ & $4.6524 E-03$ & $1.8609 E-03$ & $7.4438 E-04$ & $2.9776 E-04$ & $1.1910 E-04$ & $4.8382 E-02$ \\
40 & $2.2717 E-02$ & $9.0864 E-03$ & $3.6347 E-03$ & $1.4539 E-03$ & $5.8155 E-04$ & $2.3262 E-04$ & $9.3051 E-05$ & $3.7799 E-02$ \\
50 & $1.7747 E-02$ & $7.0990 E-03$ & $2.8396 E-03$ & $1.1358 E-03$ & $4.5434 E-04$ & $1.8174 E-04$ & $7.2694 E-05$ & $2.9530 E-02$ \\
60 & $1.2778 E-02$ & $5.1113 E-03$ & $2.0445 E-03$ & $8.1780 E-04$ & $3.2712 E-04$ & $1.3085 E-04$ & $5.2340 E-05$ & $2.1262 E-02$ \\
70 & $9.2007 E-03$ & $3.6801 E-03$ & $1.4720 E-03$ & $5.8882 E-04$ & $2.3553 E-04$ & $9.4212 E-05$ & $3.7685 E-05$ & $1.5309 E-02$ \\
80 & $6.6242 E-03$ & $2.6497 E-03$ & $1.0598 E-03$ & $4.2395 E-04$ & $1.6958 E-04$ & $6.7832 E-05$ & $2.7133 E-05$ & $1.1022 E-02$ \\
90 & $4.7695 E-03$ & $1.9078 E-03$ & $7.6311 E-04$ & $3.0524 E-04$ & $1.2209 E-04$ & $4.8839 E-05$ & $1.9535 E-05$ & $7.9361 E-03$ \\
100 & $3.4340 E-03$ & $1.3736 E-03$ & $5.4944 E-04$ & $2.1978 E-04$ & $8.7910 E-05$ & $3.5164 E-05$ & $1.4065 E-05$ & $5.7140 E-03$ \\
\hline
\end{tabular}

TABLE 2: Partial and total excited hydrogen formation cross sections $\left(\sigma_{12}\right.$ in $\left.\pi a_{0}^{2}\right)$ of proton-lithium scattering by coupled static approximation. Integration range $\left(\mathrm{IR}=32 a_{0}\right)$, number of iterations $(v=50)$ (Model I).

\begin{tabular}{lcccccccc}
\hline$k^{2} \mathrm{kev}$ & $L=0$ & $L=1$ & $L=2$ & $L=3$ & $L=4$ & $L=5$ & $L=6$ & Total \\
\hline 10 & $4.9994 E-03$ & $1.9997 E-03$ & $7.9989 E-04$ & $3.1996 E-04$ & $1.2798 E-04$ & $5.1193 E-05$ & $2.0477 E-05$ & $8.3186 E-03$ \\
20 & $3.9058 E-03$ & $1.5623 E-03$ & $6.2491 E-04$ & $2.4997 E-04$ & $9.9986 E-05$ & $3.9994 E-05$ & $1.5998 E-05$ & $6.4989 E-03$ \\
30 & $3.0513 E-03$ & $1.2205 E-03$ & $4.8821 E-04$ & $1.9529 E-04$ & $7.8114 E-05$ & $3.1246 E-05$ & $1.2498 E-05$ & $5.0772 E-03$ \\
40 & $2.3838 E-03$ & $9.5353 E-04$ & $3.8142 E-04$ & $1.5257 E-04$ & $6.1027 E-05$ & $2.4411 E-05$ & $9.7640 E-06$ & $3.9665 E-03$ \\
50 & $1.8624 E-03$ & $7.4496 E-04$ & $2.9798 E-04$ & $1.1920 E-04$ & $4.7677 E-05$ & $1.9071 E-05$ & $7.6283 E-06$ & $3.0989 E-03$ \\
60 & $1.3410 E-03$ & $5.3637 E-04$ & $2.1455 E-04$ & $8.5816 E-05$ & $3.4327 E-05$ & $1.3731 E-05$ & $5.4924 E-06$ & $2.2312 E-03$ \\
70 & $9.6543 E-04$ & $3.8619 E-04$ & $1.5447 E-04$ & $6.1790 E-05$ & $2.4716 E-05$ & $9.8864 E-06$ & $3.9545 E-06$ & $1.6064 E-03$ \\
80 & $6.9514 E-04$ & $2.7805 E-04$ & $1.1122 E-04$ & $4.4489 E-05$ & $1.7796 E-05$ & $7.1182 E-06$ & $2.8472 E-06$ & $1.1567 E-03$ \\
90 & $5.0050 E-04$ & $2.0020 E-04$ & $8.0079 E-05$ & $3.2031 E-05$ & $1.2813 E-05$ & $5.1251 E-06$ & $2.0500 E-06$ & $8.3280 E-04$ \\
100 & $3.6036 E-04$ & $1.4414 E-04$ & $5.7657 E-05$ & $2.3063 E-05$ & $9.2251 E-06$ & $3.6900 E-06$ & $1.4760 E-06$ & $5.9961 E-04$ \\
\hline
\end{tabular}

TABLE 3: Partial and total elastic cross sections $\left(\sigma_{11}\right.$ in $\left.\pi a_{0}^{2}\right)$ of proton-lithium scattering by coupled static approximation. Integration range (IR $\left.=32 a_{o}\right)$, number of iterations $(v=50)$ (Model II).

\begin{tabular}{lcccccccc}
\hline$k^{2} \mathrm{kev}$ & $L=0$ & $L=1$ & $L=2$ & $L=3$ & $L=4$ & $L=5$ & $L=6$ & Total \\
\hline 10 & $5.2934 E-02$ & $2.1174 E-02$ & $8.4694 E-03$ & $3.3878 E-03$ & $1.3551 E-03$ & $5.4204 E-04$ & $2.1682 E-04$ & $8.8079 E-02$ \\
20 & $4.1355 E-02$ & $1.6542 E-02$ & $6.6167 E-03$ & $2.6467 E-03$ & $1.0587 E-03$ & $4.2347 E-04$ & $1.6939 E-04$ & $6.8812 E-02$ \\
30 & $3.2308 E-02$ & $1.2923 E-02$ & $5.1693 E-03$ & $2.0677 E-03$ & $8.2709 E-04$ & $3.3084 E-04$ & $1.3233 E-04$ & $5.3758 E-02$ \\
40 & $2.5241 E-02$ & $1.0096 E-02$ & $4.0385 E-03$ & $1.6154 E-03$ & $6.4617 E-04$ & $2.5847 E-04$ & $1.0339 E-04$ & $4.1999 E-02$ \\
50 & $1.9719 E-02$ & $7.8878 E-03$ & $3.1551 E-03$ & $1.2620 E-03$ & $5.0482 E-04$ & $2.0193 E-04$ & $8.0771 E-05$ & $3.2811 E-02$ \\
60 & $1.4198 E-02$ & $5.6792 E-03$ & $2.2717 E-03$ & $9.0867 E-04$ & $3.6347 E-04$ & $1.4539 E-04$ & $5.8155 E-05$ & $2.3625 E-02$ \\
70 & $1.0223 E-02$ & $4.0890 E-03$ & $1.6356 E-03$ & $6.5424 E-04$ & $2.6170 E-04$ & $1.0468 E-04$ & $4.1872 E-05$ & $1.7010 E-02$ \\
80 & $7.3602 E-03$ & $2.9441 E-03$ & $1.1776 E-03$ & $4.7106 E-04$ & $1.8842 E-04$ & $7.5369 E-05$ & $3.0148 E-05$ & $1.2247 E-02$ \\
90 & $5.2994 E-03$ & $2.1198 E-03$ & $8.4790 E-04$ & $3.3916 E-04$ & $1.3566 E-04$ & $5.4266 E-05$ & $2.1706 E-05$ & $8.8179 E-03$ \\
100 & $3.8156 E-03$ & $1.5262 E-03$ & $6.1049 E-04$ & $2.4420 E-04$ & $9.7678 E-05$ & $3.9071 E-05$ & $1.5628 E-05$ & $6.3489 E-03$ \\
\hline
\end{tabular}

the number of iterations. We fix $h$ at $1 / 16$ and have obtained all the results presented below with 512 mesh points (i.e., IR = $32 a_{0}$ ). It is found that excellent convergence can be obtained with $v=50$, and this demonstrates stability of our iterative method. Final calculations have been carried out for seven partial waves corresponding to $0 \leq \ell \leq 6$ at values of $k_{1}^{2}$ representing the kinetic energy region $\left(10 \leq k_{1}^{2} \leq 1000 \mathrm{Kev}\right)$.

In Table 1, we find the partial and total elastic cross sections $\sigma_{11}$ of $p$-Li scattering (Model I). The table demonstrates that the $S$-wave scattering cross sections contribute with main part of total cross sections. The table shows also that the $\mathrm{P}$ - and D-waves are the most important partial waves after S-wave. This table emphasizes that the total elastic cross section decreases smoothly with the increase of the incident energy.

Table 2 contains the partial and total excited hydrogen, $\mathrm{H}(2 \mathrm{~s})$, formation cross sections $\sigma_{12}$ (Model I). The table illustrates that the main contribution to $\sigma_{12}$ is due to the Swave scattering cross sections. The remaining contributions to $\sigma_{12}$ are due to $\mathrm{P}$ - and $\mathrm{D}$-waves. The total excited hydrogen, 
TABLE 4: Partial and total excited hydrogen formation cross sections $\left(\sigma_{12}\right.$ in $\left.\pi a_{0}^{2}\right)$ of proton-lithium scattering by coupled static approximation. Integration range $\left(\mathrm{IR}=32 a_{o}\right)$, number of iterations $(v=50)$ (Model II).

\begin{tabular}{lcccccccc}
\hline$k^{2} \mathrm{kev}$ & $L=0$ & $L=1$ & $L=2$ & $L=3$ & $L=4$ & $L=5$ & $L=6$ & Total \\
\hline 10 & $5.8816 E-03$ & $2.3526 E-03$ & $9.4105 E-04$ & $3.7642 E-04$ & $1.5057 E-04$ & $6.0227 E-05$ & $2.4091 E-05$ & $9.7866 E-03$ \\
20 & $4.5950 E-03$ & $1.8380 E-03$ & $7.3519 E-04$ & $2.9408 E-04$ & $1.1763 E-04$ & $4.7052 E-05$ & $1.8821 E-05$ & $7.6458 E-03$ \\
30 & $3.5898 E-03$ & $1.4359 E-03$ & $5.7437 E-04$ & $2.2975 E-04$ & $9.1899 E-05$ & $3.6760 E-05$ & $1.4704 E-05$ & $5.9732 E-03$ \\
40 & $2.8045 E-03$ & $1.1218 E-03$ & $4.4873 E-04$ & $1.7949 E-04$ & $7.1796 E-05$ & $2.8719 E-05$ & $1.1487 E-05$ & $4.6665 E-03$ \\
50 & $2.1911 E-03$ & $8.7642 E-04$ & $3.5057 E-04$ & $1.4023 E-04$ & $5.6091 E-05$ & $2.2436 E-05$ & $8.9745 E-06$ & $3.6458 E-03$ \\
60 & $1.5776 E-03$ & $6.3102 E-04$ & $2.5241 E-04$ & $1.0096 E-04$ & $4.0385 E-05$ & $1.6154 E-05$ & $6.4617 E-06$ & $2.6250 E-03$ \\
70 & $1.1358 E-03$ & $4.5434 E-04$ & $1.8173 E-04$ & $7.2694 E-05$ & $2.9078 E-05$ & $1.1631 E-05$ & $4.6524 E-06$ & $1.8899 E-03$ \\
80 & $8.1781 E-04$ & $3.2712 E-04$ & $1.3085 E-04$ & $5.2340 E-05$ & $2.0936 E-05$ & $8.3743 E-06$ & $3.3497 E-06$ & $1.3608 E-03$ \\
90 & $5.8882 E-04$ & $2.3553 E-04$ & $9.4211 E-05$ & $3.7684 E-05$ & $1.5074 E-05$ & $6.0295 E-06$ & $2.4118 E-06$ & $9.7976 E-04$ \\
100 & $4.2395 E-04$ & $1.6958 E-04$ & $6.7832 E-05$ & $2.7133 E-05$ & $1.0853 E-05$ & $4.3412 E-06$ & $1.7365 E-06$ & $7.0543 E-04$ \\
\hline
\end{tabular}

TABLE 5: Present total cross sections ( $\sigma_{12}$ of Model I and Model II in $\pi a_{0}^{2}$ ) for excited hydrogen formation $(\mathrm{H}(2 \mathrm{~s}))$ with different theoretical results [5-7].

\begin{tabular}{|c|c|c|c|c|c|}
\hline$k^{2} \mathrm{kev}$ & Model I & Model II & {$[5]$} & {$[6]$} & [7] \\
\hline 50 & $3.0989 E-03$ & $3.6458 E-03$ & & $6.5 E-01$ & $3.596 E-03$ \\
\hline 60 & $2.2312 E-03$ & $2.6250 E-03$ & & $1.0 E-03$ & \\
\hline 70 & $1.6064 E-03$ & $1.8899 E-03$ & & $4.9 E-03$ & \\
\hline 80 & $1.1567 E-03$ & $1.3608 E-03$ & & $2.8 E-03$ & \\
\hline 100 & $5.9961 E-04$ & $7.0543 E-04$ & & & $6.746 E-04$ \\
\hline 150 & $1.1602 E-04$ & $1.3649 E-04$ & & & $1.294 E-04$ \\
\hline 200 & $2.5849 E-05$ & $3.0410 E-05$ & $1.048 E-05$ & & $3.294 E-05$ \\
\hline 250 & $4.3437 E-06$ & $5.1103 E-06$ & & & $1.327 E-05$ \\
\hline 300 & $2.9857 E-06$ & $3.5126 E-06$ & & & $8.489 E-06$ \\
\hline 500 & $8.0153 E-07$ & $9.4297 E-07$ & $5.212 E-07$ & & $9.828 E-07$ \\
\hline 800 & $4.2587 E-07$ & $5.0102 E-07$ & $7.711 E-08$ & & $2.278 E-07$ \\
\hline 1000 & $3.9981 E-08$ & $4.7027 E-08$ & $2.906 E-08$ & & $9.366 E-08$ \\
\hline
\end{tabular}

$\mathrm{H}(2 \mathrm{~s})$, formation cross section decreases also smoothly with the increase of $k_{1}^{2}$.

Table 3 presents the partial and total elastic cross sections $\sigma_{11}$ of Model II. The main contributions to $\sigma_{11}$ are to the $\mathrm{S}$ - and P-waves. The seven partial waves employed are quite satisfactory for calculating $\sigma_{11}$ in a high degree of accuracy within the framework of coupled static approximation.

Table 4 shows partial and total excited hydrogen, $\mathrm{H}(2 \mathrm{~s})$, cross sections $\sigma_{12}$ of $p$-Li scattering of Model II. On considering the variation of $\sigma_{12}$, with respect to incident energy, we notice that it has its maximum value at $k_{1}^{2}=10 \mathrm{Kev}$ and decreases smoothly thereafter. The values of total excited hydrogen, $\mathrm{H}(2 \mathrm{~s})$, cross sections $\sigma_{12}$ in table give larger values than of Model I.

The total elastic cross sections of proton-lithium scattering for the two models are presented in Figure 2. In Figure 3, the present total hydrogen formation cross sections of proton-lithium scattering for Model I are displayed with that of Model II. The present results keep decreasing with the increasing of energy and its values of Model II are higher than that of Model I.

Comparison between total excited hydrogen, $\mathrm{H}(2 \mathrm{~s})$, cross sections $\sigma_{12}$ of $p$-Li scattering of the two Models (I and II) and those determined by Banyard and Shirtclliffe [5], Ferrante et al. [6], and Tiwari [7] are tabulated in Table 5. Our results of Model II are found in reasonably good agreement with the above mentioned theoretical results specially with Tiwari calculations [7].

\section{Conclusions}

Proton-lithium scattering is studied using the coupled static approximation. Our interest is focused on the influence of polarization potential of the lithium atom. The switching on $V_{\mathrm{pol}}^{\mathrm{Li}(2 \mathrm{~s})}(\sigma)$ in Model II has positive influence upon total excited hydrogen formation cross sections $\sigma_{12}$. The present calculations for excited hydrogen formation cross sections, 


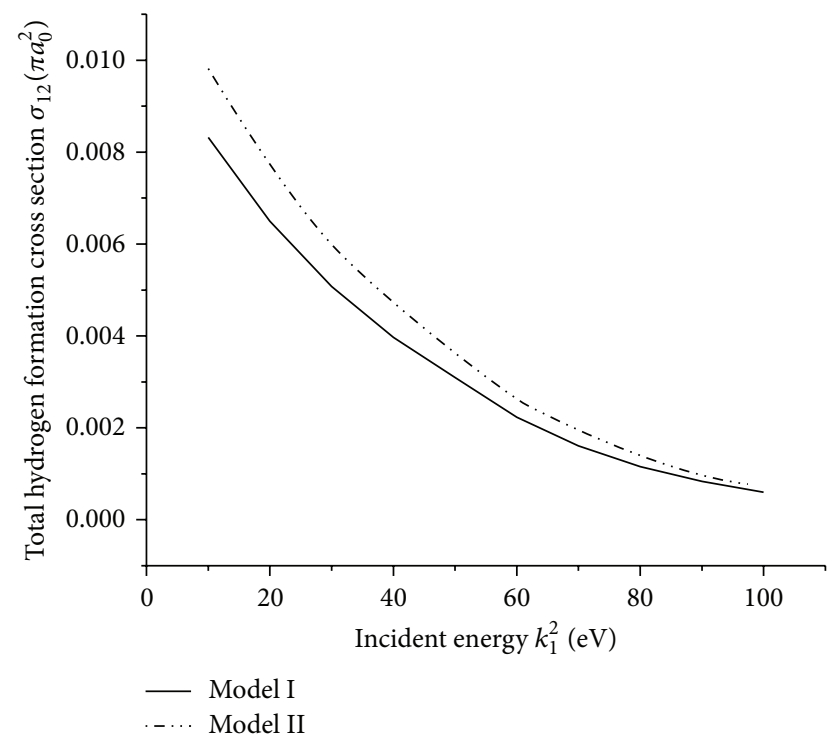

FIgure 3: Total excited hydrogen $(\mathrm{H}(2 \mathrm{~s}))$ formation cross sections $\left(\sigma_{12}\right.$ in $\pi a_{0}^{2}$ ) of proton-lithium scattering by coupled static approximation for the two models.

using Model II, show reasonable agreement with available theoretical calculations in wide energy region.

\section{Conflict of Interests}

The author declares that there is no conflict of interests regarding the publication of this paper.

\section{References}

[1] R. D. DuBois and L. H. Toburen, "Electron capture by protons and helium ions from lithium, sodium, and magnesium," Physical Review A, vol. 31, no. 6, pp. 3603-3611, 1985.

[2] W. Fritsch and C. D. Lin, "Atomic orbital expansion study of electron capture in $\mathrm{H}^{+}+\mathrm{Li}$ and $\mathrm{He}^{+}+\mathrm{Li}$ collisions," Journal of Physics B, vol. 16, no. 9, pp. 1595-1603, 1983.

[3] S. A. Elkilany, "Elastic scattering of positrons by xenon atoms," Il Nuovo Cimento D, vol. 17, no. 1, pp. 1-7, 1995.

[4] M. A. Abdel-Raouf and S. A. Elkilany, "Elastic scattering of positrons by helium, neon, argon and krypton atoms," Journal of Physics B, vol. 194, Article ID 072004, 2009.

[5] K. E. Banyard and G. W. Shirtclliffe, "Electron capture from lithium and its ions by high-energy protons," Journal of Physics B, vol. 12, no. 19, Article ID 183247, 1979.

[6] G. Ferrante, E. Fiordilino, and M. Zarcone, "Valence and core electron capture in proton-alkali atom collisions," Il Nuovo Cimento B, vol. 52, no. 2, pp. 151-164, 1979.

[7] Y. N. Tiwari, "Formation of H-atom in 2s excited state of protonlithium and proton-sodium scattering," Pramana: Journal of Physics, vol. 70, no. 4, pp. 753-758, 2008.

[8] K. Basuchoudhury and D. P. Sural, "Electron capture in ground and excited states in proton-alkali-metal-atom collisions," Journal of Physics B, vol. 25, p. 853, 1992.

[9] M. Horbatsch, J. W. Darewych, and R. P. McEachran, "Polarised density approach to atomic collisions. Scattering of positrons from atomic hydrogen and the noble gases," Journal of Physics $B$, vol. 16 , no. 23 , pp. 4451-4465, 1983.

[10] E. W. McDaniel and M. R. C. McDowell, "Case studies in atomic collision physics," in Polarized Orbital Approximations, R. J. Drachman and A. Temkin, Eds., vol. 2, chapter 6, NorthHolland, Amsterdam, The Nertherlands, 1972.

[11] S. A. Elkilany, "Formation of antihydrogen in antiproton positronium inelastic scattering," Indian Journal of Physics, vol. 88, no. 1, pp. 19-24, 2014.

[12] E. Clementi and C. Roetti, "Roothaan Hartree Fock atomic wavefunctions. Basis functions and their coefficients for ground and certain excited states of neutral and ionized atoms, $\mathrm{Z} \leq 54$," Atomic Data and Nuclear Data Tables, vol. 14, no. 3-4, pp. 177478, 1974.

[13] G. Peach, "Model potentials or pseudopotentials; what's the difference?" Comments Atomic and Molecular Physics, vol. 11, pp. 101-118, 1982.

[14] S. A. Elkilany, "Collision of positrons with positroniums," International Journal of Pure and Applied Physics, vol. 5, p. 101, 2009. 

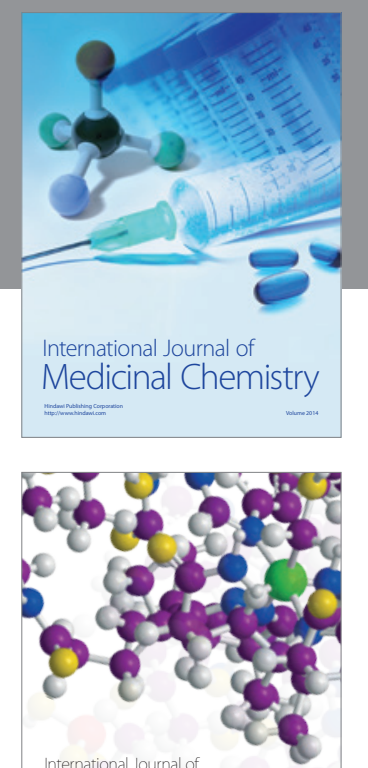

\section{Carbohydrate} Chemistry

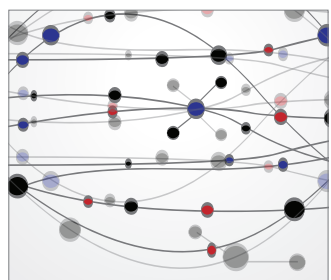

The Scientific World Journal
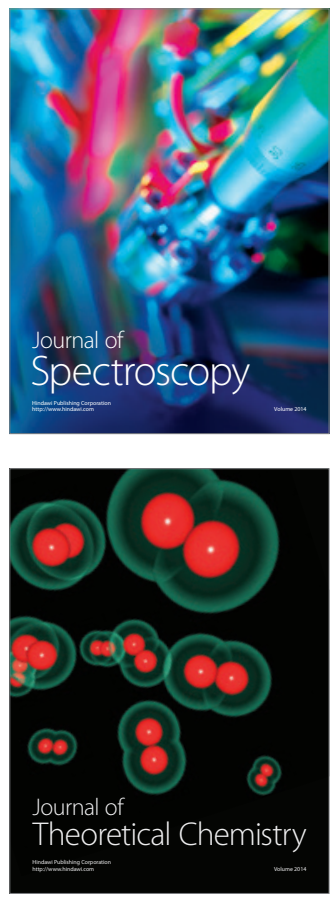
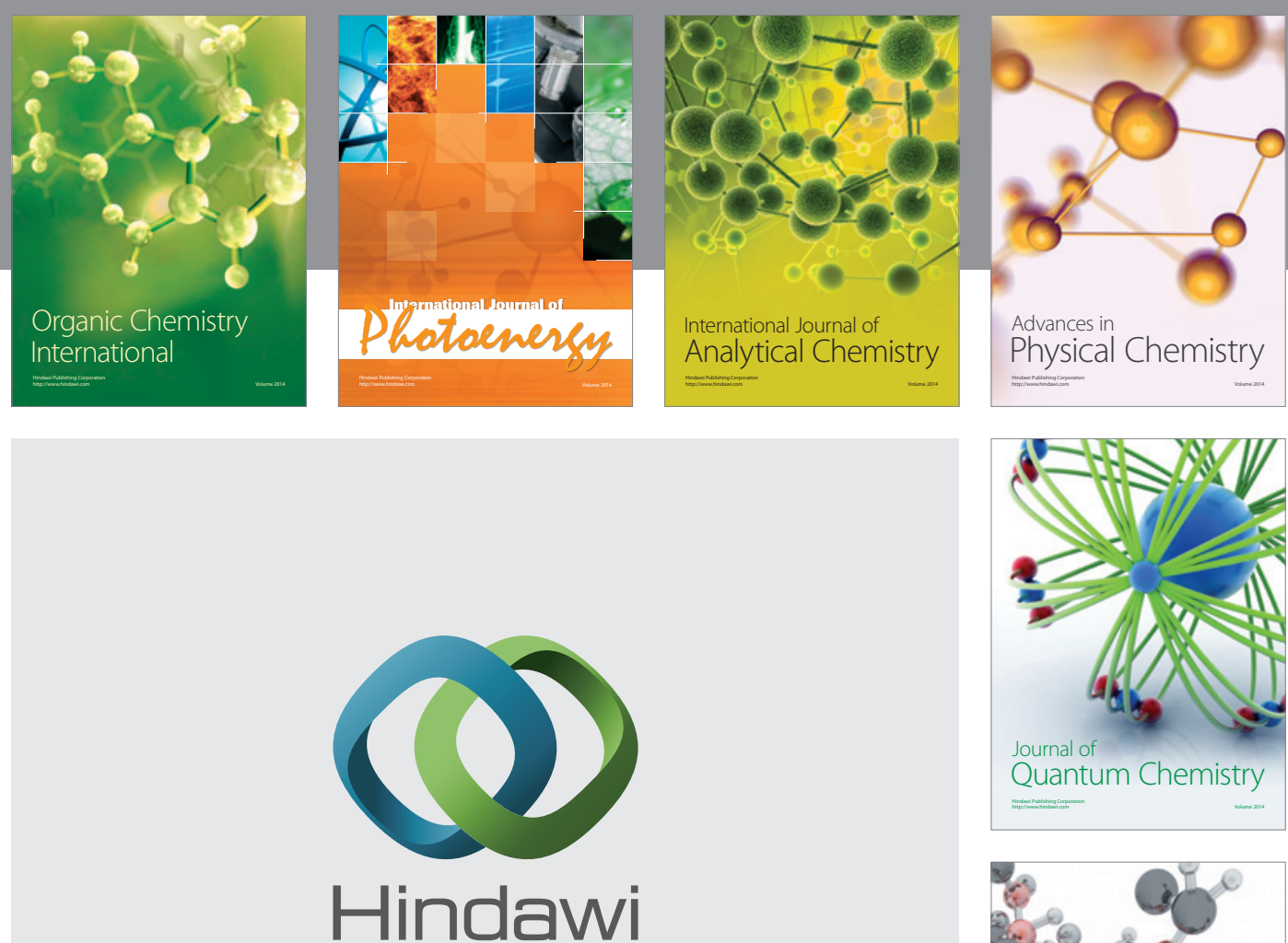

Submit your manuscripts at

http://www.hindawi.com

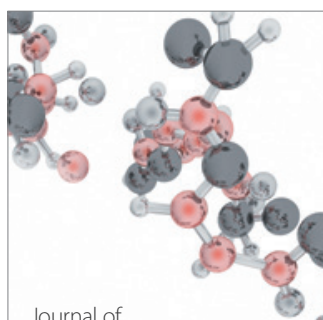

Analytical Methods

in Chemistry

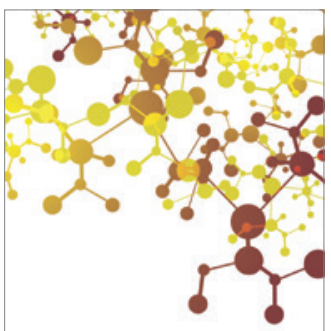

Journal of

Applied Chemistry

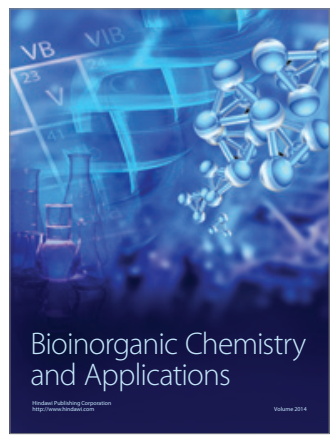

Inorganic Chemistry
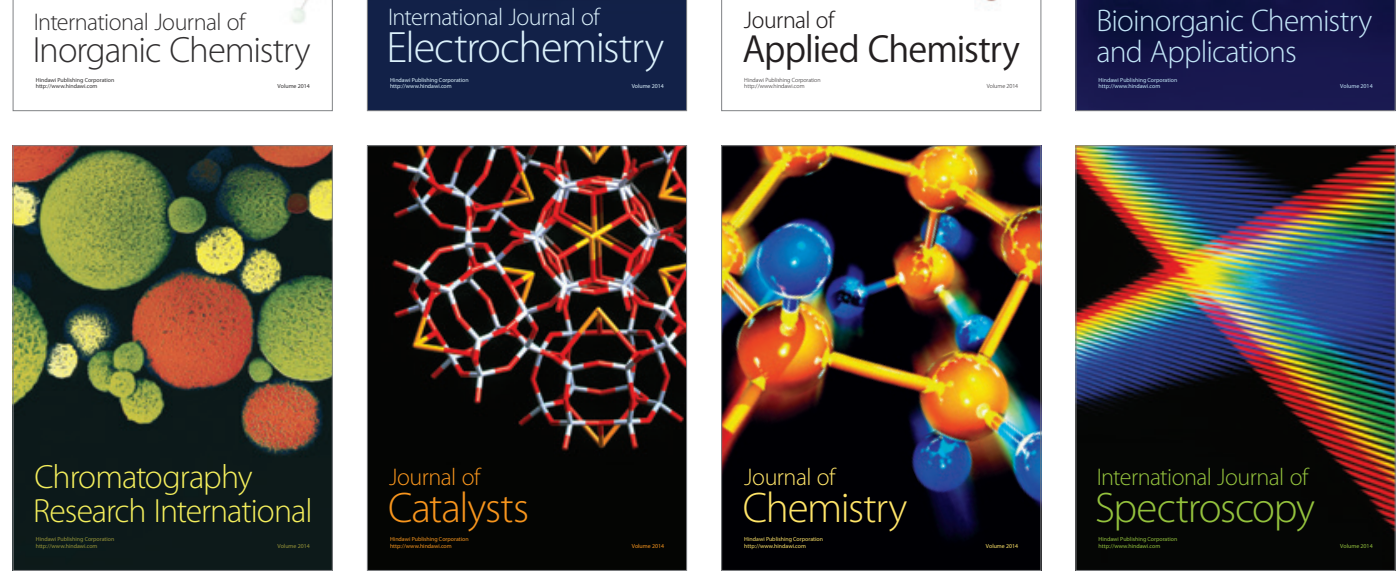